\title{
Presentación. El crecimiento expansivo metropolitano en el sur global: un proceso transformacional sui géneris.
}

\author{
Eduardo Sousa-González ${ }^{1}$
}

Es evidente que toda manifestación que involucra el proceso que se vincula con la transformación ciudadmetrópoli, como una evolución urbana en la esfera mundial, es progresivo, creciente y seguramente irreversible; ya lo mencionaba hace décadas Henry Lefebvre (1980, 2013, 2017), donde pronosticaba la "urbanización completa de la sociedad" (1980, 2013, 2017). Desde luego, estaremos de acuerdo en que lo que se visualiza en el futuro inmediato y de largo plazo, es poco probable que se produzca alguna transformación espacial que tienda a prescindir de este tipo sui géneris de concentraciones urbanas en el sur global e invariablemente en el mundo conocido.

En este sentido, la evidencia empírica ha demostrado que una de las particularidades que puntualiza a esta evolución transformacional ciudadmetrópoli, es aquella que tiende a mostrar fases secuenciales en el proceso de urbanización, las cuales no sólo están asociadas con la concentración creciente de pobladores que tienden a aglutinarse en espacios físicos definidos; sino también, la manera particular en que se concentran los empleos que involucran a los sectores secundario y terciario a partir de un centro metropolitano; constituyéndose así, en agrupaciones de concentraciones demográficas dominantes con múltiples limitantes de política pública, que puedan incidir positivamente en un control eficiente, efectivo y eficaz (EEE) de su hinterland espacial de orientación periférica.

No obstante, estas grandes concentraciones de población, de actividades productivas, de equipamientos de salud, educativos, lúdicos, de infraestructura básica y complementaria y demás, han adquirido en estos tiempos líquidos (Bauman, Z. 2009; Bachelard, G: 2005) una importancia de orden cuantitativo y cualitativo revelador (Sousa, E.:2008, 2010); ya que la evidencia mostrada en las grandes metrópolis del sur global, indican procesos espaciales de expansión periférica similares, todos ellos enmarcados en la teoría que está vinculada a las etapas de metropolización de base poblacional y de base empleos secundarios y terciarios; todo esto basado en el concepto propuesto denominado ciclo reproductor de la vida de los pobladores, el cual se representa en el diagrama 1.

La evidencia histórica en las metrópolis mexicanas y seguramente las del sur global, indican que es a partir del establecimiento físico de un asentamiento humano en una ciudad central, donde al evolucionar el ciclo reproductor de la vida de los pobladores y al activarse la movilidad social espacial de estos y la saturación de suelo urbano inicial; es donde se forma una nueva demanda de superficie urbana, gestándose el desplazamiento de habitantes hacia zonas periféricas inmediatas o de proximidad relativa; muchas de las veces sin una planeación eficiente, efectiva y eficaz de parte de las autoridades gubernamentales; permeando en todo este proceso de ocupación espacial y de expansividad territorial, dos variables que están concatenadas en una correspondencia biunívoca: Por un lado:

i. El grado de desarrollo del lugar;

ii. Su gobernabilidad;

iii. La característica de la infraestructura

instalada y de los equipamientos ofrecidos.

Por el otro:

i. El grado de desarrollo económico de la zona;

ii. Fundamentado en la característica de la oferta de empleos vinculados al sector secundario y terciario; que, dicho sea de paso, este último, es el de más amplio espectro de desarrollo en la contemporaneidad. Así se representa en el diagrama $1(c f r$.$) .$

\footnotetext{
${ }^{1}$ Nacionalidad: mexicano; Adscripción: Facultad de Arquitectura de la Universidad Autónoma de Nuevo León, México; doctor en Filosofía con Orientación en Arquitectura y Asuntos Urbanos; miembro del Sistema Nacional de Investigadores (CONACYT) ratificado en el Nivel 2; miembro de la Academia Mexicana de Ciencias AMC desde el año 2008; eduardo.sousagn@uanl.edu.mx

${ }^{2}$ Para profundizar en estos conceptos se recomienda consultar Sousa, E. (2015). De la ciudad a la metrópoli. Nuevas realidades territoriales. Chile; Trama.
} 
Diagrama $\mathrm{N}^{\circ} 1$ : Proceso iterativo de metropolización; basado en

la velocidad del ciclo reproductor de la vida de los pobladores

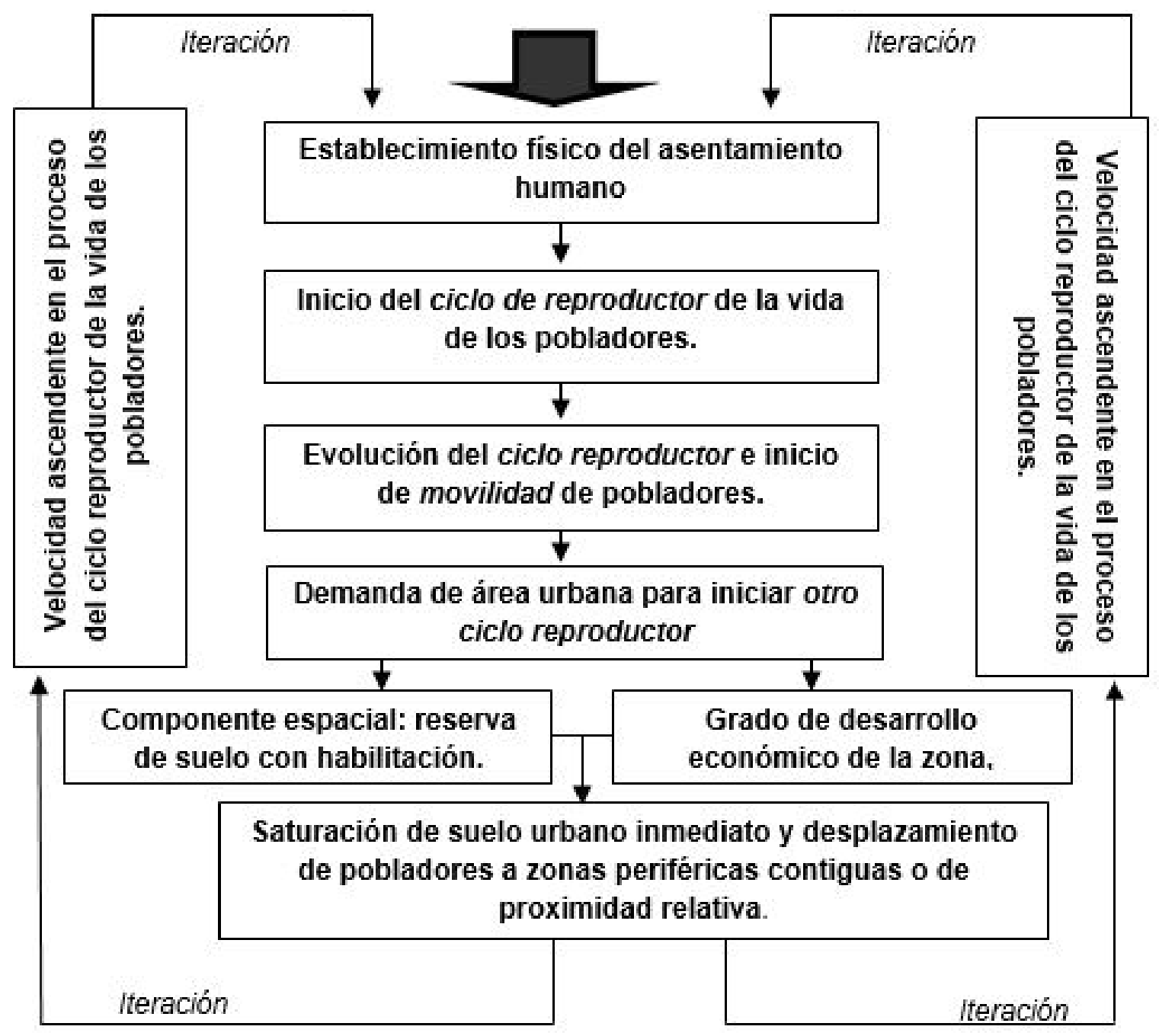

Fuente: datos generados por Sousa, E. 2008

Estos procesos transformacionales de crecimiento expansivo demográfico, basados en los ciclos reproductores de la vida de los pobladores de las ciudades, tienen una relación dual basada en una correspondencia que se denomina en esta investigación como centro-periferia:

1. El centro representado por el centro metropolitano o la ciudad central de la conurbación;

2. La periferia, formada por las áreas urbanas de los municipios conurbados procesalmente en un período de tiempo y entre éstas dos, se desarrolla toda la compleja vida en una sociedad metropolitana; según se explica en la figura 1.
Figura 1. Crecimiento expansivo metropolitano

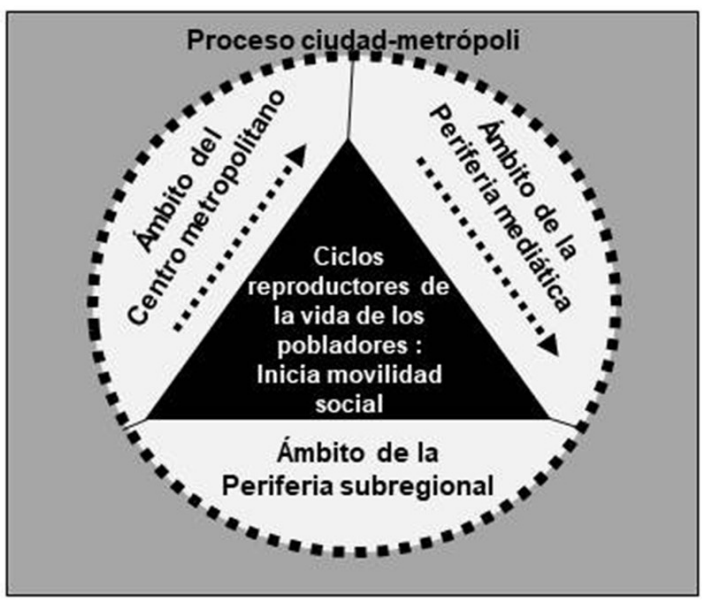

Fuente: datos generados en esta investigación 
Entonces, sería posible esbozar en forma preliminar una primicia en cuanto a que la evolución de los ciclos reproductores mencionados, estarían representados por el crecimiento y reproducción de pobladores y son los que caracterizan finalmente al proceso de metropolización de las ciudades; el cual está indicado en la teoría de las etapas de metropolización, por las que atraviesan la mayoría de las metrópolis desarrolladas o subdesarrolladas del mundo; las cuales algunos autores como: Busquets, (1993:165), Sobrino (2003:198) y otros; las simbolizan con cuatro procesos plenamente identificables:

1. Urbanización: cuando la tasa de crecimiento porcentual anual de pobladores de la ciudad central supera a la de la periferia. 2. Suburbanización: cuando la periferia alcanza una mayor tasa de crecimiento porcentual anual.

3. Desurbanización: cuando la cuidad central observa un despoblamiento relativo o absoluto.

4. Reurbanización: cuando en la ciudad central ocurre un repoblamiento relativo o absoluto.

Desde esta perspectiva que involucra a las ciudades: sus procesos de crecimiento expansivo, su arquitectura sus formas de diseño y sus múltiples variables intervinientes; la doctora Hermida Rubio Gutiérrez Facultad de Arquitectura de la Universidad Veracruzana Xalapa, nos indica que el artículo que presenta se centra en exponer lo establecido personas teóricas, críticas y hacedoras de arquitectura, para desarrollar una manera de leer (entender) y escribir (construir) la ciudad: la narrativa, el arte de contar historias. Es necesaria para explicar el mundo, para construir la memoria y para proyectar hacia el futuro. Está inmersa a medio camino entre el mundo tangible y el imaginario, además es un proceso práctico, simbólico y emotivo a la vez. Así, la narrativa se entiende epistemológicamente, como una manera de comprender el mundo y la vida. Con base en este argumento, se profundiza sobre el entendimiento de la ciudad. Se observan además varios caminos que han retomado estas ideas o han experimentado otras nuevas, y finalmente se demuestra que la narrativa tiene mucho fundamento desde la teoría, la historia y la crítica de la arquitectura; sin embargo, desde la arquitectura y el urbanismo como disciplinas, no ha tenido la suficiente aplicación, ni se ha considerado seriamente como enfoque.
Por su parte, la doctora Rubí del Rocío Vázquez Cruz de la Universidad del Valle de Atemajac, Querétaro, presenta una investigación sobre las zonas metropolitanas de México, indicando que los efectos del proceso de metropolización han generado un crecimiento urbano de tipo disperso. Se conoce que este fenómeno emerge en el contexto de la globalización, como un proceso socioeconómico que se manifiesta físicamente en el territorio y produce transformaciones urbanas, ambientales, económicas, sociales, políticas y culturales en las ciudades. Aplicando el enfoque de análisis territorial, el estudio aborda el proceso de metropolización en la Zona Metropolitana de Querétaro, la cual ha destacado por loacelerado que se ha expandido en su superficie de urbanización. En este caso, la metodología muestra la expansión secuencial de la urbanización en un territorio de escala metropolitana y como puede explorarse comparativamente entre las décadas 1990-2010.

En el caso de los doctores Arturo Ojeda de la Cruz, Clara Rosalía Álvarez Chaves y David Carlos Orona Llano exponen los resultados de una investigación sobre un estudio que se desarrolló con motivo del recurrente problema de inundación que se presenta en la zona urbana de Hermosillo en temporada de lluvias, y en el principal campus de la universidad de Sonora. El estudio consistió en la evaluación del sistema de drenaje pluvial en el campus universitario mediante la aplicación de herramientas y principios hidrológicos, hidráulicos, topográficos y sociales para determinar los caudales que genera el escurrimiento del agua pluvial en las doce microcuencas identificadas, derivado del proceso estadístico de las lluvia máximas diarias de una serie de anual de 34 años de registro climático en la estación meteorológica del centro de la ciudad. Se obtuvieron las curvas de precipitación-duración-periodo de retorno (P-dTr) eligiendo una tormenta de diseño para un periodo de retronó de 10 años, y una duración de la tormenta igual al tiempo de concentración que fue variable para cada una de la microcuencas. Una vez definidos los caudales se calcularon los elementos hidráulicos necesarios para captar y conducir el agua pluvial; finalmente se estableció la modalidad para aprovechar el agua en la gestión del agua de lluvia atendiendo un aprovechamiento sostenible.

Los doctores Erle Garcia Estrada, Jesús Enrique De Hoyos Martínez y Víctor Daniel Ávila 
Akerberg de la Universidad del Estado de México, abordan la temática sobre el cuidado ambiental, indicando que éste transita incongruentemente entre la destrucción y contaminación; y el cuidado y conservación, en específico en las ciudades. La consecuencia de la destrucción y contaminación es que propicia la modificación casi irreversible del metabolismo ambiental al interior de las urbes, al provocar la aparición de vulnerabilidades en el sistema natural que producen desechos o salidas nocivas para el ambiente y para la población que habita en ellas. El cuidado y la conservación, procura el restablecer el balance en el metabolismo ambiental citadino. Este artículo busca conservar las áreas verdes urbanas como una posibilidad de restablecer el equilibrio en el metabolismo ambiental de las ciudades. Método utilizado, es la discusión teórica de los conceptos de metabolismo, metabolismo ambiental, cuidado, conservación y áreas verdes urbanas es el contraste del pensamiento y análisis de diversos autores. La contribución es definir el concepto de metabolismo ambiental, además de generar conciencia hacia el cuidado y conservación de las áreas verdes urbanas a través de un marco conceptual y un esquema vinculado.

Los doctores María Erandi Flores Romero investigadora de la Universidad Nacional Autónoma de México; Irving Omar Morales Agiss jefe del departamento de análisis de datos en Digital State y Liliana Beatríz Sosa Compeán de la Facultad de Arquitectura de la Universidad Autónoma de Nuevo León (UANL), presentan los resultados de una investigación en donde se propone un método para identificar estructuras dentro de una red de vialidades con un algoritmo de detección de comunidad basado en flujos, implementado en un grafo que representa la red de vialidades de la ciudad. De acuerdo con los resultados obtenidos en las ciudades de México y Monterrey, el método divide efectivamente la infraestructura vial en varias comunidades y preserva la vecindad geográfica. Las fronteras de las comunidades coinciden con las divisiones administrativas junto con otras fronteras dentro de la ciudad. La identificación de comunidades podría ser útil para estudiar la heterogeneidad de la conectividad de las calles dentro de la ciudad, lo que podría conducir a mejoras en la movilidad urbana o incluso a la aplicación de políticas públicas.

En el caso del doctor Avatar Flores Gutiérrez de la Universidad Autónoma de Querétaro en su artículo nos indica que: A pesar de la diversidad de aproximaciones a la conceptualización en el diseño arquitectónico, la idea de que el concepto atiende a una analogía formal está aún extendida. La analogía formal del concepto suele estar vinculada únicamente a aspectos simbólicos de la composición para el observador y no a la esencia de lo descubierto en el proceso de investigación y análisis proyectual. Desde la perspectiva del fenómeno arquitectónico como sistema, esta idea provoca un rompimiento entre la investigación proyectual y la forma, y preserva la dicotomía forma-función. En este artículo se aborda la idea de que el proceso de conceptualización en arquitectura puede ser abordado desde una perspectiva sistémica del fenómeno arquitectónico fundamentada en la noción de autopoiesis de Maturana y Varela, de los sistemas de Christopher Alexander, y la investigación de Flores-Gutiérrez sobre el fenómeno arquitectónico. El texto concluye con la idea de que, al dejar fuera al concepto como guía de composición formal y adoptar un proceso de conceptualización sistémica orientado a la definición de un sistema generador, el diseñador puede ampliar los límites de su investigación para buscar no sólo la configuración y relaciones adecuadas para la actividad, sino la atmósfera necesaria para dichas actividades.

Por último, la doctora Eugenia M. VillalobosGonzález de la Facultad de Arquitectura y Urbanismo, Universidad Central de Venezuela, nos expone una visión sobre la arquitectura indicando que ésta ha sido muy estudiada desde los estilos arquitectónicos, los arquitectos con sus obras representativas, y el dilema de su vinculación con el arte y la ciencia, pero poco desde el ejercicio profesional. Por ello, se presentan los resultados de una investigación documental sobre la práctica de arquitectura y su compromiso con la sociedad, a través de la revisión de: (1) el binomio arquitectura-proyecto, (2) la evolución de la profesión en el tiempo (3) los retos contemporáneos de la profesión de cara a la sociedad (4) la construcción social como práctica inclusiva de los diversos interesados. $\mathrm{La}$ arquitectura procura espacios para personas y comunidades que, dada la complejidad de las edificaciones, también se construye con personas que se agrupan para lograr un objetivo común, la edificación. Existen muchas críticas que señalan la arquitectura como una profesión elitista y formalista, entonces el reto del arquitecto contemporáneo está vinculado a asumir su rol social 
como facilitador de la construcción del proyecto a través de la participación activa y comprometida de los interesados; esta es la construcción social del proyecto que reconoce el papel de cada uno de los actores con sus contribuciones y limitaciones.

Como comentario final, los miembros del equipo que conforman la Revista CONTEXTO. Revista de la Facultad de Arquitectura de la Universidad Autónoma de Nuevo León, estamos plenamente convencidos que el investigador y el lector común interesado en la diversa temática expuesta, encontraran a través de las páginas que componen este proyecto editorial, un tópico con una visión interdisciplinar, de actualidad e internacional; enfocada en diferentes esferas del quehacer investigativo, en donde, investigadores de alta calificación, han colaborado con sus trabajos personales o grupales en la generación de este número.

Seguramente los artículos que se incluyen aquí serán no solamente de interés, sino también de utilidad concreta en futuras investigaciones, complaciendo al investigador y al lector más exigente.

Finalmente, es necesario reconocer que lo expuesto a través de este número de CONTEXTO, es un esfuerzo continuado a lo largo de muchos años por mantener una calidad de excelencia editorial, que ha sido compartido por autores, pares de evaluadores, el Comité Editorial, la Coordinación Editorial, el equipo de apoyo y por supuesto, las autoridades institucionales que forman la Facultad de Arquitectura, su Posgrado y la Universidad Autónoma de Nuevo León. C

\section{BIBLIOGRAFÍA.}

Bachelard, G. (2005). El agua y los sueños. México; FCE.

Bauman, Z.(2008). Modernidad Liquida. México, FCE.

Lefebvre, Henry. (1980). La revolución urbana. Madrid; Alianza

Lefebvre, Henry. (2013). El derecho a la ciudad. Madrid; Capitán Swing

Lefebvre, Henry. (2017). La producción del espacio. Madrid; Capitán Swing

Sousa, E. (2015). De la ciudad a la metrópoli. Nuevas realidades territoriales. Chile; Trama.

Sousa, E. (2008). Los componentes de las fuerzas centrífugas como elementos generatrices de los contornos metropolitanos. Revista Urbano, Chile. $\mathrm{N}^{\circ} 018$ (12), Julio-diciembre 2008.
Sousa, E. (2008). El proceso de ensanchamiento urbano-periférico como un fenómeno espacio-temporal ligado a la diferenciación espacial: ciudad-metrópoli. Revista Entelequia, España. № 12, otoño 2010. 